and mortality were recorded. The performance of the models was assessed using standardised mortality ratios (SMR) of observed vs. predicted outcomes for calibration, and concordance (c)-statistics for discrimination.

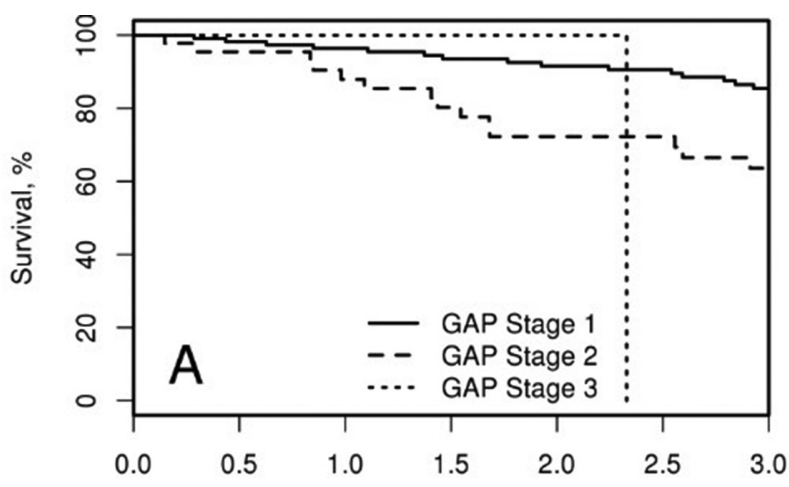

Years since ILD diagnosis

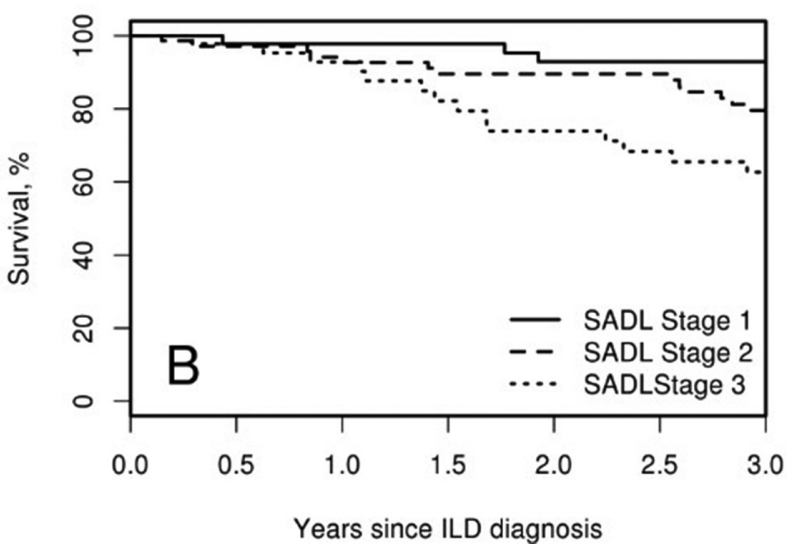

Results: The cohort included 179 patients with SSc-ILD. Mean age at ILD diagnosis was 57.8 years. There was a female predominance $(73 \%)$. The mean length of follow up after ILD diagnosis was 4.3 years (SD 4.0). Based on high resolution chest CT (or biopsy when available), $147(83 \%)$ were characterised as nonspecific interstitial pneumonia (NSIP), 31 (17\%) as usual interstitial pneumonia (UIP), 1 as unclassifiable ILD. A history of smoking was noted in $49 \%$, and this was not associated with ILD pattern (former/current $46 \%$ in NSIP vs. $61 \%$ in UIP, $p=0.11$ ). Pulmonary hypertension (PHTN) was noted in $66(49 \%)$ at baseline, and this did not differ between ILD subtypes. $84 \%$ had limited cutaneous SSc, $9 \%$ had diffuse cutaneous, and 7\% SSc sine scleroderma. SSc specific serologies (i.e., SCL-70, centromere, and/or RNA Pol III) were positive in 73 (43\%) patients, somewhat more common in NSIP than UIP ( $47 \%$ vs $26 \%$; $p=0.034)$. During a median of 3.2 years of follow-up, 65 patients died. SSc-ILD patients with UIP had a higher mortality than those with NSIP (hazard ratio: 2.27 ; 95\% Cl: 1.03-4.97). Other risk factors for progression included baseline DLCO $(p<0.001)$, FVC $(p<0.001)$ and PHTN $(p=0.012)$. All 3 models had comparable discrimination $(c=0.72,0.72$, and 0.70 , respectively). Figure 1 shows the differential mortality based on the GAP and SADL staging systems. (Note the staging in the GAP and ILD-GAP models are identical.) Regarding calibration, the ILD-GAP model underestimated mortality (SMR: 1.5; 95\% Cl: 1.05-2.14). Calibration was acceptable for SADL (SMR: 0.77; 95\% Cl: 0.54-1.10) and GAP (SMR: 0.90; 95\% Cl: 0.63-1.29). The SADL model overestimated mortality in Stage III ILD.

Conclusions: The ILD-GAP model underestimated mortality, and the SADL model overestimated mortality in certain subgroups. However, the GAP model performed well in this cohort, providing the best prognostic information for SScILD.
REFERENCES:

[1] Ley B, et al. Annals of internal medicine 2012;156(10):684-691.

[2] Ryerson CJ, et al. Chest 2014;145(4):723-728.

[3] Morisset J, et al. Chest 2017;152(5):999-1007.

Disclosure of Interest: None declared

DOI: 10.1136/annrheumdis-2018-eular.3331

\section{FRI0434 PATIENTS WITH INFLAMMATORY MYOPATHIES ADMITTED IN ICU ARE CHARACTERISED BY RECENT ONSET AND UNTREATED ACTIVE DISEASE AS WELL AS OLDER AGE AND HIGH COMORBIDITIES}

B. Michard, T. Artzner, F. Severac, M. Schenck, J.-E. Herbrecht, R. JanssenLangenstein, O. Collange, G. Freys, J. Pottecher, J. Sibilia, T. Martin, F. Meziani, F. Schneider, A. Meyer. CHU Strasbourg, Strasbourg, France

Background: Inflammatory myopathies (IM) are life-threatening but treatable diseases. The risk factors for admission in Intensive care unit (ICU), the management and the outcome of patients with IM admitted to ICU has not yet been assessed.

Objectives: To assess the clinical features, risk factors and outcome of patients with IM admitted in ICU.

Methods: A single centre cohort of 509 patients with IM was screened for admission in ICU from 1992 to 2017. Patients admitted for trauma or for complications from elective surgery were excluded. Control patients with IM who had not been hospitalised in ICU were randomly selected from the cohort.

Results: Thirty-two ICU admissions were recorded in $27 \mathrm{IM}$ patients during the study period $(<0.05 \%$ of admissions over this 25 year period). Three IM patients were admitted more than once.

Characteristics and prognosis of patients in ICU

Patients hospitalised in ICU had a mean age of $63 y+ \pm 15$ with SAPS II score of 58 \pm 24 and LODS score of $9 \pm 5$ corresponding to an intermediate severity at admission in ICU. The delay between IM diagnosis and first ICU admission was $27 \pm 43$ months. It is noteworthy that 12 patients $(44 \%)$ were admitted in the ICU within the first month of IM diagnosis, among whom 4 (15\%) were diagnosed with IM during ICU stay. Sixteen patients $(60 \%)$ were not treated at the time of their first ICU admission. In $56 \%$ of the ICU stays, patients had active disease at admission. Patients were most frequently admitted for respiratory failure $(88 \%)$ but cardiac $(47 \%)$, renal $(47 \%)$, neurologic $(47 \%)$, haematological $(22 \%)$ and hepatic $(15 \%)$ failures were also recorded. Infections were present in $72 \%$ of the ICU stays, with septic shock in $44 \%$. Nine patients (33\%) died in ICU and 3 others (11\%) within 90 days of the last ICU discharge (vs. $15 \%$ during a $7.5 \pm 5$ years period of follow-up in the control group, $p<0.0001$ ).

Risk factors of ICU admission: The case control analysis identified 6 risk factors significantly associated with hospitalisation in the ICU, 3 of which were independently associated with hospitalisation in multivariate analysis. ICU patients had a higher age at first clinical signs of illness $(62 \pm 13$ years old vs. $53 \pm 13$ years old, $p<0.05)$, they had a higher rate of chronic kidney failure ( $26 \%$ vs. $0 \%, p<0.05)$ and higher incidence of arterial or venous thrombosis history $(37 \%$ vs. $0 \%$, $\mathrm{p}<0.05)$. Other risk factors that were only identified in univariate analysis included lower BMI (22.6 \pm 4.5 vs. $25.4 \pm 6.3)$, a history of interstitial lung disease ( $48 \%$ vs. $30 \%$ ) and a higher Charlson comorbidity index (4.6 \pm 2.6 vs. $3.3 \pm 2$ ).

The type of myositis was not significantly associated with admission in ICU, although no patient admitted in ICU had sIBM. It is noteworthy that cumulative number of immunomodulatory treatments in patients at the time of hospitalisation in ICU was lower than in the control group ( $0.5 \pm 0.7$ vs. $1.9 \pm 0.8 p<0.001)$.

Conclusions: IM patients admitted in ICU frequently have recent onset and untreated active IM with respiratory failure. Admission to ICU is associated with older age and a higher number of comorbidities. Mortality of IM patients in ICU is high.

Disclosure of Interest: None declared

DOI: 10.1136/annrheumdis-2018-eular.6148 